\title{
Material Dependence of Multiaxial Low Cycle Fatigue Damage under Non-proportional Loading
}

\author{
Takamoto ITOH $^{1}$ and Tao YANG ${ }^{2}$ \\ ${ }^{1}$ Division of Mechanical Engineering, Graduate School of Engineering, \\ University of Fukui \\ 9-1, Bunkyo 3-chome, Fukui-shi, Fukui 910-8507, Japan \\ Fax:+81-(0)776-27-8748, E-mail: itoh@u-fukui.ac.jp \\ ${ }^{2}$ Graduate Student, Graduate School of Engineering, University of Fukui
}

\begin{abstract}
This study discusses evaluation of material dependence of multiaxial low cycle fatigue ( $L C F)$ to develop a suitable strain parameter for life estimation under non-proportional loading. It has been reported that fatigue lives are reduced accompanying an additional hardening under strain controlled non-proportional loading in which principal directions of stress and strain are changed in a cycle. Strain controlled multiaxial LCF tests using proportional and non-proportional strain paths were carried out using hollow cylinder specimens of several materials. The reduction in low cycle fatigue life due to non-proportional loading is discussed relating to the additional cyclic hardening behaviors and its material dependence. Material constant, $\alpha$, used in strain parameter for life estimation under non-proportional multiaxial LCF is also discussed.
\end{abstract}

\section{INTRODUCTION}

Components and structures like pressure vessels and high temperature exchangers undergo low cycle fatigue (LCF) damage. In multiaxial LCF under strain controlled non-proportional loading in which principal directions of stress and strain are changed in a cycle, it has been reported that fatigue lives are reduced accompanying with an additional hardening which depends on both strain paths and materials [1-5]. Thus, developing an appropriate design parameter for multiaxial LCF is required for the reliable designs and maintenances of structure components.

Itoh et al. [4-7] have carried out a series of multiaxial LCF tests under non-proportional loading with various strain paths combined axial and shear loadings using a hollow cylinder specimen and have examined the dependence of the life on the strain path and the material. They also proposed a strain parameter for estimating multiaxial LCF life under non-proportional loading. However, these studies were performed mainly for materials of which crystal structure is face-centered cubic lattice (FCC), but no study for materials with other crystal structures like body-centered cubic lattice (BCC). Thus, it should be necessary to examine the fatigue life properties for the 
Table 1. List of materials and parameters employed.

\begin{tabular}{|c|c|c|c|c|c|c|c|c|}
\hline Material & $\mathrm{CS}$ & $A$ & $B$ & $\alpha$ & $\alpha^{*}$ & $\sigma_{\mathrm{B}}$ & $\sigma_{0.2}$ & $\left(\sigma_{\mathrm{B}}-\sigma_{0.2}\right) / \sigma_{\mathrm{B}}$ \\
\hline SUS316 & \multirow{6}{*}{ FCC } & 0.010 & 0.93 & 0.75 & 0.75 & 575 & 260 & 0.55 \\
\hline SUS304 & & 0.012 & 0.87 & 0.9 & 0.8 & 750 & 290 & 0.61 \\
\hline SUS304 (923K) & & 0.011 & 0.14 & 0.40 & 0.52 & 480 & 130 & 0.73 \\
\hline SUS310S & & 0.009 & 0.92 & 0.76 & 0.70 & 520 & 215 & 0.59 \\
\hline OFHC & & 0.009 & 0.16 & 0.16 & 0.20 & 240 & 182 & 0.24 \\
\hline 6061Al & & 0.018 & 0.16 & 0.41 & 0.48 & 390 & 253 & 0.35 \\
\hline SGV410 & \multirow{5}{*}{$\mathrm{BCC}$} & 0.008 & 0.85 & 0.39 & 0.85 & 470 & 275 & 0.41 \\
\hline $\mathrm{S} 25 \mathrm{C}$ & & 0.008 & 0.49 & 0.28 & 0.65 & 493 & 354 & 0.28 \\
\hline $\mathrm{S} 45 \mathrm{C}$ & & 0.011 & 0.78 & 0.22 & 0.50 & 630 & 445 & 0.29 \\
\hline $\mathrm{S} 55 \mathrm{C}$ & & 0.012 & 0.48 & 0.25 & 0.45 & 695 & 485 & 0.25 \\
\hline SUS430 & & 0.009 & 0.68 & 0.28 & 0.65 & 480 & 263 & 0.45 \\
\hline
\end{tabular}

materials which show the different deformation behaviors [10] and to discuss the applicability of the strain parameter for life estimation.

In this study, multiaxial LCF tests under strain controlled proportional and non-proportional loading were carried out using hollow cylinder specimens of several kinds of materials to examine the relationship between additional hardening and reduction in failure life due to non-proportional loading. This study also discusses the material constant, $\alpha$, used in the strain parameter for life estimation under non-proportional multiaxial LCF and proposes a simple method to revaluate $\alpha$ using material constant resulted from monotonic tension test.

\section{MATERIALS AND TEST PROCEDURE}

Test materials employed were various metallic materials of which crystal structures are face-centered cubic lattice (FCC) or body-centered cubic lattice (BCC) as listed in Table 1. In the table, coefficients $A$ and $B$, parameters $\alpha$ and $\alpha^{*}$, etc. will be mentioned later. The specimen used was a hollow cylinder specimen with O.D. $12 \mathrm{~mm}$, I.D. $9 \mathrm{~mm}$ and G.L. $7 \mathrm{~mm}$ as shown in Fig. 1.

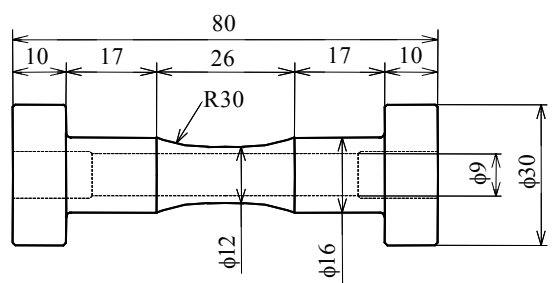

Figure1. Shape and dimensions of specimen (mm). 


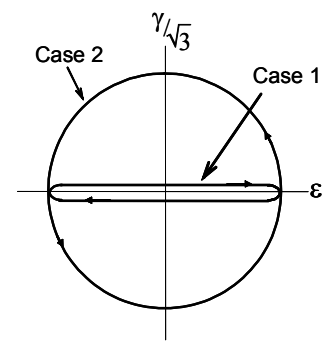

Figure 2. Strain paths employed.

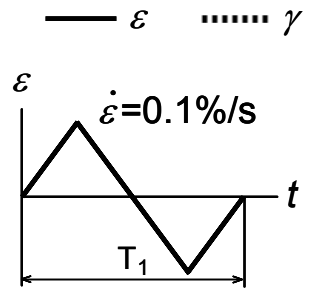

Case 1

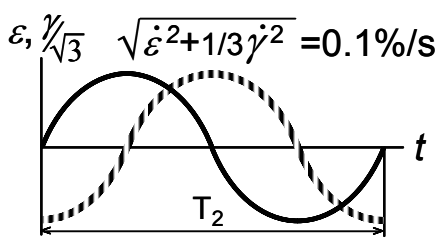

Case 2
Figure 3. Axial and shear strain waveforms.

Total strain controlled multiaxial LCF tests were conducted under 2 types of strain paths. Figures 2 and 3 show the strain paths on $\varepsilon-\gamma / \sqrt{3}$ plot and the strain waveforms of $\varepsilon$ and $\gamma$, respectively, where $\varepsilon$ and $\gamma$ are axial and shear strains. Case 1 is the push-pull test and Case 2 the $90^{\circ}$ sinusoidal out-of-phase loading test. The former is the proportional loading test and the later the non-proportional loading test. Total strain ranges were set to the same ranges in Case 1 and Case 2 and strain rate was $0.1 \% / \mathrm{sec}$ based on Mises basis. Number of cycles to failure (failure life), $N_{\mathrm{f}}$, is determined as the cycles at which axial or shear stress range was reduced to $3 / 4$ from that at $1 / 2 N_{\mathrm{f}}$.

\section{RESULTS AND DISGUSIONS}

\section{Strain Parameter for Life Estimation under Non-proportional Loading}

In multiaxial LCF of austenite stainless steels, failure lives decrease drastically under non-proportional loading accompanied a large additional hardening if the data are correlated by Mises' total equivalent strain range. It has been reported that the large reduction in life has a close relation with strain paths and materials. Therefore, Itoh et al. [4-7] proposed the non-proportional strain range for life estimation under non-proportional loading as equated,

$$
\Delta \varepsilon_{\mathrm{NP}}=\left(1+\alpha f_{\mathrm{NP}}\right) \Delta \varepsilon \mathrm{I}
$$

where $\Delta \varepsilon \mathrm{I}$ is the maximum principal strain range under non-proportional loading which can be calculated by $\varepsilon$ and $\gamma . \alpha$ and $f_{\mathrm{NP}}$ are the material constant and non-proportional factor, respectively. The former is the parameter related to the additional hardening due to non-proportional loading and the later is the parameter expressing the intensity of non-proportional loading.

The value of $\alpha$ can be given by two methods [10]. One method is to define $\alpha$ as the ratio of increase in stress amplitude in Case 2 to that in Case 1. The other method is to define $\alpha$ as $N_{\mathrm{f}}$ in Case 2 becomes the life equivalent to $N_{\mathrm{f}}$ in Case 1 at the same $\Delta \varepsilon$ I. $f_{\mathrm{NP}}$ is defined as, 


$$
f_{\mathrm{NP}}=\frac{\mathrm{k}}{\mathrm{T} \varepsilon \mathrm{I}_{\max }} \int_{0}^{\mathrm{T}}(|\sin (\xi(\mathrm{t}))| \varepsilon \mathrm{I}(\mathrm{t})) \mathrm{dt}, \quad \mathrm{k}=\frac{\pi}{2}
$$

where $\mathrm{T}$ is the time for a cycle, $\mathrm{k}$ is a constant for making $f_{\mathrm{NP}}=1$ in the circular straining on $\varepsilon-\gamma / \sqrt{3}$ plot and $\mathrm{k}=\pi / 2$. $\varepsilon \mathrm{I}(\mathrm{t})$ is the maximum absolute value of principle strain given by $\varepsilon \mathrm{I}(\mathrm{t})=\operatorname{Max}\left[\left|\varepsilon_{1}(\mathrm{t})\right|,\left|\varepsilon_{3}(\mathrm{t})\right|\right]$ at time $\mathrm{t}$ and the $\varepsilon_{\max }$ is the maximum value of $\varepsilon \mathrm{I}(\mathrm{t})$ in a cycle. In the equation, the angle $\xi(\mathrm{t}) / 2$ is employed in order to describe the rotation of principal strain direction, Then, $\xi(\mathrm{t})$ is the angle between $\varepsilon \mathrm{I}_{\max }$ and $\varepsilon \mathrm{I}(\mathrm{t})$ and has double amplitude compared with that in the specimen [6]. The integrand measures the rotation of the maximum principal strain direction and the integration of strain amplitude after the rotation. Therefore, $f_{\mathrm{NP}}$ totally evaluates the severity of non-proportional straining in a cycle.

\section{Evaluation of Multiaxial LCF Life}

To discuss the material dependence of life, this section shows multiaxial LCF properties of SUS316 and SGV410. Figures 4 (a) and (b) show $N_{\mathrm{f}}$ correlated by non-proportional strain range, $\Delta \varepsilon_{\mathrm{NP}}$. In the figures, the bold line was drawn based on the data of Case 1 and the two thin lines show a factor of 2 band. The material constant, $\alpha$, employed here is determined by evaluating the degree of additional hardening. For SUS316 $(\alpha=0.75)$ in Fig. 4 (a), $N_{\mathrm{f}}$ in Case 2 is almost the same as that in Case 1. On the other hand, $N_{\mathrm{f}}$ in Case 2 for SGV410 $(\alpha=0.39)$ in Fig. 4 (b) is correlated unconservatively. The similar trend can also be observed in other FCC and BCC materials.

The difference of properties of reduction in life and additional hardening due to non-proportional loading may come from the difference of slip mechanism between FCC and BCC materials. Indeed, crack initiation and growth behaviors under

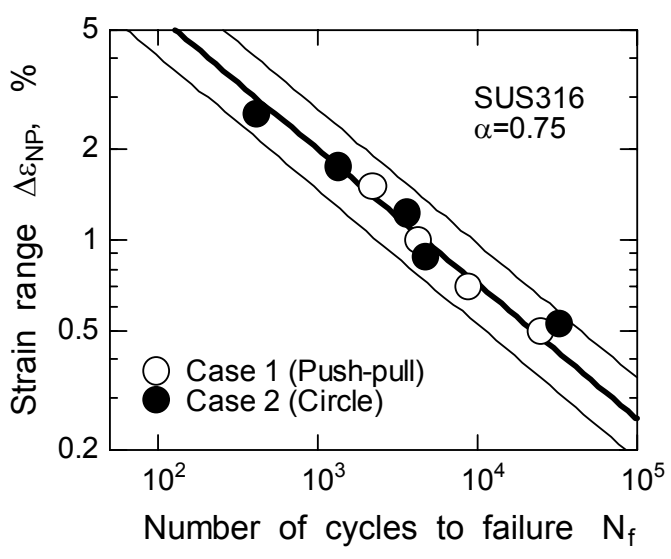

(a) SUS316

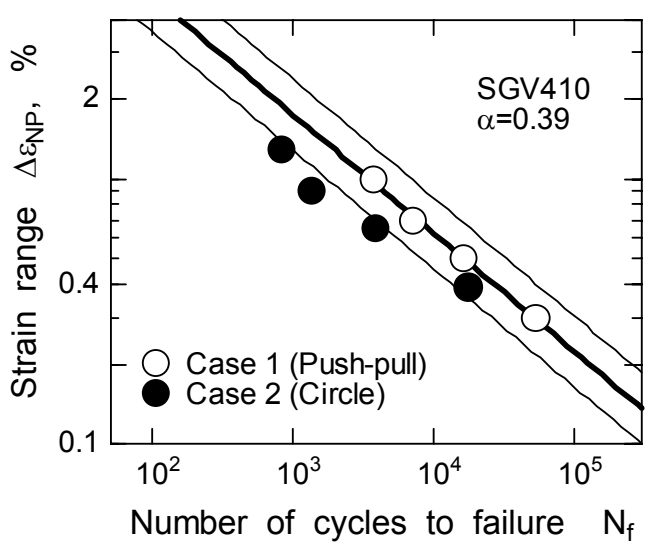

(a) SGV410

Figure 4. Relationship between $\Delta \varepsilon_{\mathrm{NP}}$ and $N_{\mathrm{f}}$. 


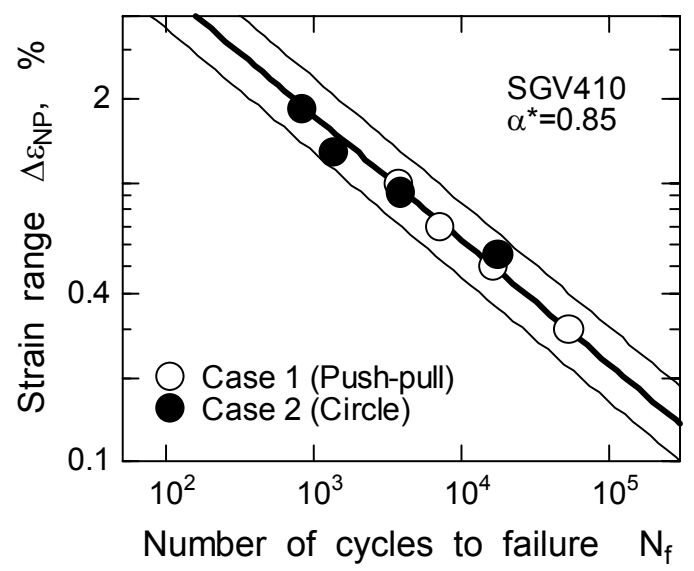

Figure 5. Relationship between $\Delta \varepsilon_{\mathrm{NP}}$ and $N_{\mathrm{f}}$ for SGV410 with $\alpha=0.85$.

proportional and non-proportional loadings are different, but more detail description which can be referred to previous Itoh's work [7] is omitted here due to a limitation of maximum number of page.

Figure 5 shows the re-plot of relationship between $\Delta \varepsilon_{\mathrm{NP}}$ and $N_{\mathrm{f}}$ for SGV410 by using $\alpha^{*}$ as material constant for evaluating the degree of reduction in life. Correlation in Fig. 5 shows that $N_{\mathrm{f}}$ in Case 2 can be correlated within the factor of 2 band with $\alpha^{*}=0.85$.

\section{Evaluation of Material Constant $\alpha$}

In order to investigate the relationship between properties of multiaxial LCF life and additional hardening under non-proportional loading, this section evaluates the relationship between the material constants $\alpha$ and $\alpha^{*}$ used in the non-proportional strain range, $\Delta \varepsilon_{\mathrm{NP}}$, based on the results obtained by tested materials.

To obtain life curves with a small number of data in Case 1 and Case 2 for each material, the universal slope method was employed equated in Eq. 3 [11].

$$
\Delta \varepsilon_{\mathrm{NP}}=\left(1+\alpha^{*} f_{\mathrm{NP}}\right) \Delta \varepsilon \mathrm{I}=A N_{\mathrm{f}}^{-0.12}+B N_{\mathrm{f}}^{-0.6}
$$

where the coefficients $A$ and $B$ are equated as $3.5 \sigma_{B} / E$ and $\varepsilon_{\mathrm{f}}^{0.6}$, respectively according to the definition of the universal slope method. Here, $E, \sigma_{\mathrm{B}}$ and $\varepsilon_{\mathrm{f}}$ are Yong's modulus, strength and elongation. In this study, $A$ is put as $3.5 \sigma_{\mathrm{B}} / E$ accordingly, but $B$ is determined as life curves based on the lives in Case 1. $\alpha^{*}$ is put as lives in Case 1 and Case 2 correspond at the same $\Delta \varepsilon I$ for each material. In this study, monotonic tension test were conducted for each material to obtain these material constants including $0.2 \%$ proof stress, $\sigma_{0.2}$. The constants obtained from the test were listed on Table 1.

Figure 6 shows the relationship between $\alpha$ and $\alpha^{*}$ for each material. The solid mark shows the data for BCC materials and the open mark for FCC materials. Keys shown in 


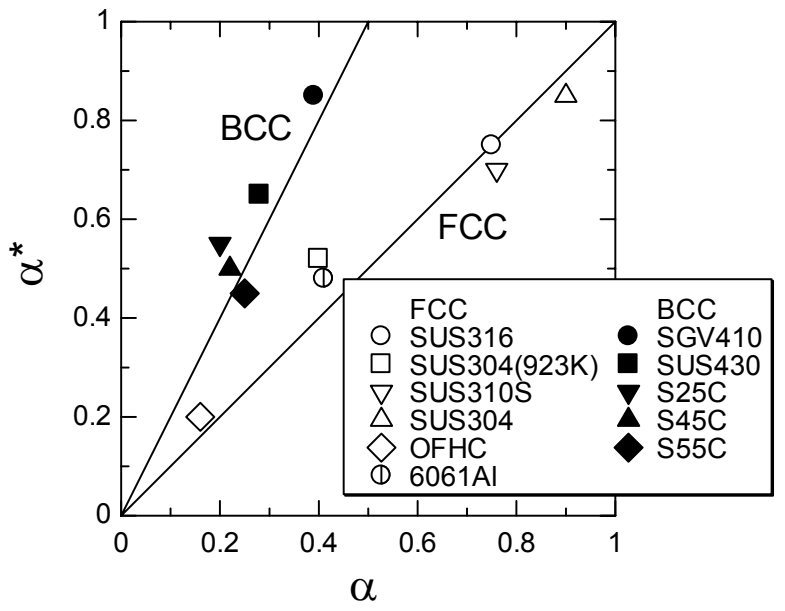

Figure 6. Relationship between $\alpha$ and $\alpha^{*}$.

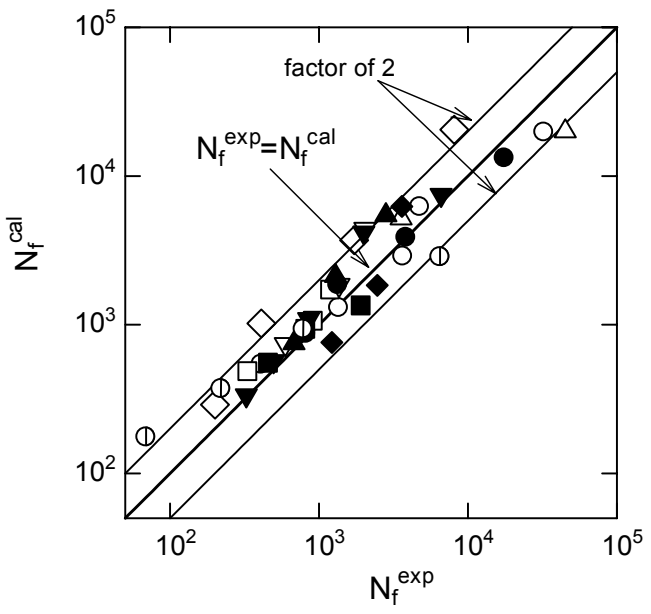

Figure 7. Comparison of $N_{\mathrm{f}}$ between calculation and experiment.

the figure will be referred in the following figures. The relationship is shown by two straight lines for FCC and BCC materials separately although a few data are scattered slightly. It suggests that reduction in life has closely relationship with additional hardening in non-proportional loading, which depends on crystal structure of tested materials. The relationship between $\alpha$ and $\alpha^{*}$ can be expressed experimentally as,

$$
\alpha^{*}=\left\{\begin{array}{ccc}
\alpha & \text { for } & \mathrm{FCC} \\
2 \alpha & \text { for } & \mathrm{BCC}
\end{array}\right.
$$

In order to verify the application of life estimation under non-proportional loading, the comparison of $N_{\mathrm{f}}^{\exp }$ and $N_{\mathrm{f}}^{\text {cal }}$ in Case 2 is shown in Fig. 7. $N_{\mathrm{f}}^{\text {exp }}$ is the life obtained from experiment and $N_{\mathrm{f}}^{\text {cal }}$ the life evaluated by Eq. 1 based on life curve in push-pull test. In the calculation by Eq. $1, \alpha^{*}$ is used for material constant. All the data are correlated within the factor of 2 band, which suggests that failure lives under non-proportional loading for various materials can be estimated by $\Delta \varepsilon_{\mathrm{NP}}$ if the degree of additional hardening is known with using the relationship in Eq. 4.

\section{A SIMPLE METHOD FOR EVALUATION OF $\alpha$ AND LIFE EVALUATION}

As discussed above, multiaxial LCF life shows the large reduction in life under non-proportional loading in comparison with that under proportional loading. By using non-proportional strain parameter, $\Delta \varepsilon_{\mathrm{NP}}$ in Eq. 1, multiaxial LCF life can be estimated from the data in push-pull loading test. However, to obtain the value of material constant, $\alpha$, multiaxial fatigue tests under non-proportional loading is necessary, but it is usually difficult to conduct. If $\alpha$ can be obtained without conducting the multiaxial 


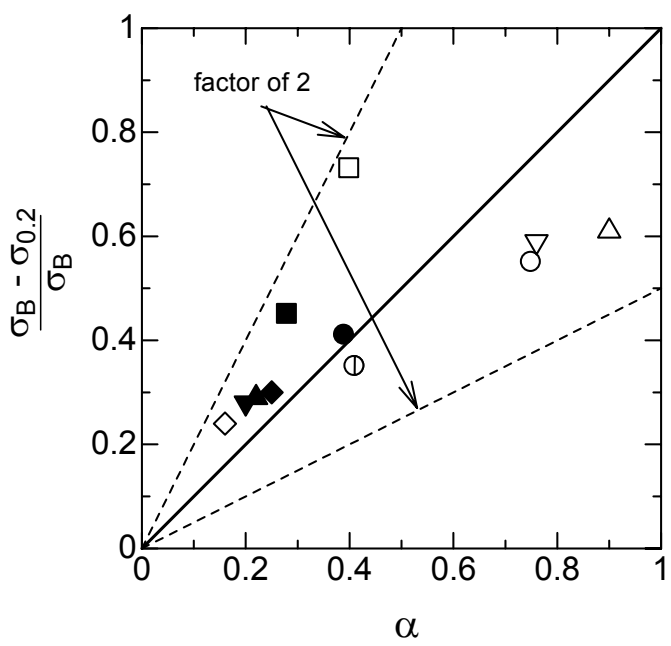

Figure 8. Relationship between $\left(\sigma_{\mathrm{B}}-\sigma_{0.2}\right) / \sigma_{\mathrm{B}}$ and $\alpha$.

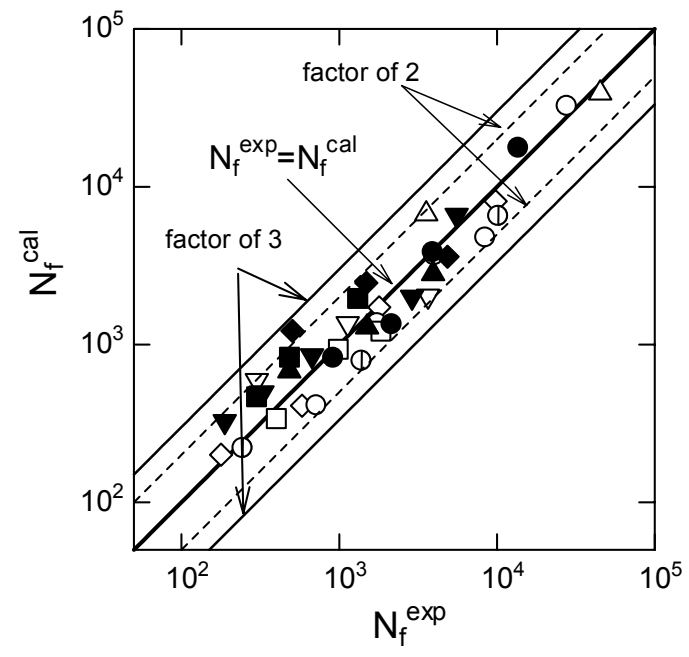

Figure 9. Comparison of $N_{\mathrm{f}}$ between experiment and calculation by Eq. 6.

fatigue test, it will be very convenient for engineers to estimate LCF life under non-proportional loading.

This section discusses the revaluation of $\alpha$ with focusing on the relationship between $\alpha$ and material constants resulted from the monotonic tension test. Cyclic hardening and additional hardening behaviors should have close relationship with static deformation behavior, then relationship between $\left(\sigma_{\mathrm{B}}-\sigma_{0.2}\right) / \sigma_{\mathrm{B}}$ and $\alpha$ is shown in Fig. 8. Although some scatter of data can be seen, but the relationship can be equated approximately as,

$$
\alpha=\frac{\left(\sigma_{\mathrm{B}}-\sigma_{0.2}\right)}{\sigma_{\mathrm{B}}}
$$

According to Eqs 1, 4 and 5, non-proportional strain range, $\Delta \varepsilon_{\mathrm{NP}}$, can be rewritten as,

$$
\Delta \varepsilon_{\mathrm{NP}}^{\prime}=\left(1+\mathrm{K} \frac{\sigma_{\mathrm{B}}-\sigma_{0.2}}{\sigma_{\mathrm{B}}} f_{\mathrm{NP}}\right) \Delta \varepsilon \mathrm{I}
$$

where coefficient $\mathrm{K}$ takes $\mathrm{K}=1$ for $\mathrm{FCC}$ materials and $\mathrm{K}=2$ for $\mathrm{BCC}$ materials.

Figure 9 shows the comparison of $N_{\mathrm{f}}$ in Case 2 between experiment and calculation. In the figure, $N_{\mathrm{f}}^{\text {exp }}$ is the life in experiment and $N_{\mathrm{f}}^{\text {cal }}$ the life estimated based on life curve in push-pull test (Case 1 test) with using $\Delta \varepsilon_{\mathrm{NP}}$ in Eq. 6 . Consequently, all the data is correlated within the factor of 3 band and most of them correlated within the factor of 2 band. The good correlation in Fig. 9 indicates that multiaxial LCF life under non-proportional can be evaluated by Eq. (6) and material constants resulted from monotonic tension test. 


\section{CONCLUSIONS}

1. Reduction in life has closely relationship with additional hardening under non-proportional loading which depends on crystal structure of tested materials.

2. $\alpha$ and $\alpha^{*}$ which related to reduction in life and additional hardening due to non-proportional loading are revaluated and can be equated by different linear relationships in BCC and FCC materials, respectively.

3. $\alpha$ has a closely relation with the behavior of monotonic tension test and $\alpha$ can be equated as $\alpha=\left(\sigma_{\mathrm{B}}-\sigma_{0.2}\right) / \sigma_{\mathrm{B}}$. The failure lives in non-proportional loading can be estimated by $\Delta \varepsilon_{\mathrm{NP}}$ where $\alpha$ is replaced by $\left(\sigma_{\mathrm{B}}-\sigma_{0.2}\right) / \sigma_{\mathrm{B}}$.

\section{REFERENCES}

1. Nitta, A., Ogata, T. and Kuwabara, K., (1989) J. Soc. Mat. Sci., Japan, Vol.36, pp.376-382. (in Japanese)

2. Doong, S.H., Socie, D.F. and Robertson, I.M., (1990) Trans. ASME, JEMT., Vol.112, pp.456-464.

3. Wang, C.H. and Brown, M.W., (1993), Fatigue and Fracture of Engng. Mater. Struct., 16, No.12, pp.1285-1298.

4. Itoh, T., Sakane, M., Ohanami, M. and D.F. Socie, (1995) Trans. ASME JEMT, Vol.117, pp.285-292

5. Itoh, T., T. Nakata, Sakane, M. and Ohnami, M., (1999) Multiaxial Fatigue and Fracture (Macha et al., eds.), ESIS-25, pp.41-54.

6. Itoh, T., (2001) J. Soc. Mat. Sci., Japan, 50, No.12, pp.1317-1322.

7. Itoh, T., Murashima, K. and Hirai, T., (2007) J. Soc. Mat. Sci., Japan, 56, pp.157-163. (in Japanese)

8. Doong, S.H., Socie, D.F., (1991) Trans. ASME, JEMT., 113, No.1, pp.23-30.

9. Itoh, T., Sakane, M., Ohanami, M. and Ameyama, (1992) K., J. Soc. Mat. Sci., Japan, 41, No.468, pp.1361-1367.

10. Itoh, T., Sakane, Hata, T. and Hamada, N., (2006) IJF, 28, No.5-6, pp.495-466

11. Manson, S.S., (1965) Exp. Mech. 5, pp.193-226. 